Article

\title{
Space Charge Measurement and Modelling in Cross-Linked Polyethylene
}

\author{
Yunpeng Zhan ${ }^{1, *} \mathbb{0}$, George Chen ${ }^{1, *} \mathbb{B}$, Miao Hao ${ }^{1}, \mathrm{Lu} \mathrm{Pu}^{2}$, Xuefeng Zhao ${ }^{2}$, Sen Wang ${ }^{2}$ and \\ Jian Liu ${ }^{2}$ \\ 1 Electrical Power Engineering Research Group, University of Southampton, Southampton SO17 1BJ, UK \\ 2 State Grid Shaanxi Electric Power Research Institute, Xi'an 710100, China \\ * Correspondence: yz10n16@soton.ac.uk (Y.Z.); gc@ecs.soton.ac.uk (G.C.); Tel.: +44(0)-23-8059-5166 (Y.Z.)
}

Received: 10 March 2020; Accepted: 5 April 2020; Published: 13 April 2020

\begin{abstract}
Cross-linked polyethylene, commercially known as XLPE, is widely used as an insulating material in high voltage cables. However, space charge accumulation under the DC field is one of the most challenging problems in the further development of XLPE insulated cable. Due to the potential electrical degradation ageing process triggered by the accumulated space charges, the IEEE standard 1732 was established for measuring space charge in HVDC extruded cables as the qualification tests. Previous research has revealed that space charge originates from either charge injection at the electrodes or ionization of impurities presenting inside the bulk. In the light of this, this paper aims to simulate the accumulation of space charge in XLPE under DC stress. Space charge measurements have been carried on the fresh and degassed XLPE samples. A modified bipolar charge transport model, by considering the dissociation of impurities, has been employed to simulate the space charge behavior in XLPE. Compared with the experimental observations, the simulation results can reveal appropriate features of hetero charge formation. Both the calculated charge dynamics and field variation are consistent with the experiment results. The restrictions and potential improvements of this preliminary model are also discussed for its future application of XLPE cables.
\end{abstract}

Keywords: cross-linked polyethylene; space charge measurement; ionization; bipolar charge transport model; hetero charge formation

\section{Introduction}

High voltage direct current (HVDC) power transmission systems have been developed rapidly in recent decades, as this technology contributes to both large-capacity power transmission over long distance and the interconnection of regional power grids [1,2]. As the main component of the HVDC transmission, a number of HVDC cable technologies have been developed and deployed successfully around the world. Currently, although the majority of the well-established HVDC cable systems in operation are oil-filled (OF) or mass-impregnated (MI) cable systems, the HVDC extruded cable attracts more interest, due to the factors of higher conductor temperature, easier installation and maintenance, and less environmental impact [3]. However, it is well reported that space charge accumulation under DC stress could distort the electric field distribution in cross-linked polyethylene (XLPE) insulated cable $[4,5]$. By creating zones of high electrical field, space charge formation can accelerate the dielectric ageing process and even lead to early electrical failure [6,7]. Thus, measurement, understanding, and modelling of space charge processes are essential, in order to calculate the electric field distribution in DC cables for a service lifetime estimation.

Cross-linked polyethylene exhibits a desirable combination of properties for being applied as the main insulation of power cables. XLPE is widely used in HVAC cable systems, and now it has been also successfully applied in HVDC systems since the development of High Voltage Self-commutated Voltage 
Converter (VSC) technology. Although it has been proposed that polypropylene can potentially replace cross-linked polyethylene as the main extruded cable insulation in future, the polypropylene-based power cable is still not a fully established technology in DC systems yet [8]. It has been verified that space charge in XLPE may originate from the charge injection at the electrodes, or from the ionization process of some dissociable chemical species, such as crosslinking by-products, antioxidants, and other impurities [9]. Many researchers were dedicated to study the space charge behaviour in polymeric insulation materials by using space charge measurement techniques, such as the pulsed electro-acoustic method (PEA) $[10,11]$. Takeda et al. reported that the hetero charges were formed due to the presence of thermally dissociated antioxidant, and the crosslinking by-products, such as acetophenone, also assisted the hetero charge formation [12]. Sekii et al. also found that the combination of acetophenone and moisture is responsible for hetero charge creation [13]. The impact of the presence of different crosslinking by-products has been studied individually when they are soaked in polyethylene, and it was found that cumyl alcohol could introduce a greater amount of space charges [14]. With the observation of fast charge packets in XLPE samples under high DC field (over $20 \mathrm{kV} / \mathrm{mm}$ ), it can be debated that the blocked extraction of injected charge carriers also assists the hetero charge formation [15]. However, it is still difficult to distinguish whether the hetero charges are partially blocked charge carriers at the extraction electrodes or dissociated ions, as the space charge measurement results only reveal net charge density.

Modelling and simulation of space charge can assist to comprehend and analyze material behavior. A macroscopic conductivity-based model has been an important reference for HVDC cable design, and actually started with the work of McAllister et al. [16]. By assuming the volume conductivity of insulation depends on temperature and field, such a model can simulate either a positive or a negative charge accumulation inside the bulk. However, the limitations of such a model have been recognized, as the complicated conduction mechanisms are neglected, and it cannot distinguish between the electronic and ionic charges [17]. Since the work proposed by Alison et al. on charge transport modelling in polyethylene-based material, the bipolar charge transport theory has been successfully applied to simulate charge dynamics in low density polyethylene (LDPE) [18]. Some emphasis have been put on simulating the space charge accumulation in XLPE flat samples, or even in cable geometry by considering the restricted charge extraction, but the contribution from ionization process is rarely mentioned [19-21].

In this paper, firstly, space charge measurements are carried out on LDPE and XLPE samples, in order to observe and compare the homo charge and hetero charge formation. After that, a bipolar charge transport model and the modified model are briefly introduced, and utilized to simulate the space charge behavior in LDPE and XLPE separately, by considering the ionization. Comparisons of the experimental and simulated results are made. Finally, the feasibility of the modified model is discussed when modelling the space charge and electric field distribution in HVDC cable insulation.

\section{Experimental Methods}

Space charge distributions in three kinds of samples, including additive-free LDPE film, fresh, and degassed XLPE flat samples, were measured under an applied DC field of $20 \mathrm{kV} / \mathrm{mm}$ at room temperature $\left(25^{\circ} \mathrm{C}\right)$, by employing the PEA measurement system. The top and bottom electrode is semi-conductive material and aluminum respectively. LDPE has been chosen as the reference in this study, because it is not only the base material for XLPE, and there are no crosslinking by-products or other impurities able to dissociate. A DC field of $20 \mathrm{kV} / \mathrm{mm}$ has been chosen, as it is not only higher than the threshold field at which the charge injection takes place in polymers, but also suitable to better observe the generation of ions. Additionally, it is also the desired average electric field in the further development of DC cables. The thickness of all the prepared samples is about $180 \mu \mathrm{m}$. The measurement has been repeated three times on each kind of sample to ensure accuracy and validity. 


\subsection{Sample Preparation}

The XLPE pellets used in this study were produced from the Borlink ${ }^{\mathrm{TM}}$ Technology, which had already been mixed with $2 \%$ Dicumyl peroxide (DCP), and crosslinking was performed by using hot press. The initial temperature was set at $120{ }^{\circ} \mathrm{C}$ for $5 \mathrm{~min}$, and then the temperature of $180{ }^{\circ} \mathrm{C}$ was applied to activate the crosslinking process for $10 \mathrm{~min}$. The mechanical pressure of 5 tons was maintained continuously during the cooling down process, and the samples were removed from the hot press once $100{ }^{\circ} \mathrm{C}$ was reached and cooled naturally down at room temperature. In this study, degassing of the XLPE samples was achieved in a vacuum oven at $80^{\circ} \mathrm{C}$ for 3 days, with the aim of expelling volatile residual by-products, and to highlight any influence this may have on the material performance. The samples were then cooled down to room temperature and sealed in polyethylene bags, prior to tests.

\subsection{Space Charge Measurement}

The space charge behavior in LDPE film is shown in Figure 1. The obvious homo charge distribution is clearly observed within the bulk. A large amount of positive charge carriers penetrated from the anode and moved towards to the cathode, while the accumulation of negative charge carriers near the cathode was fairly limited.

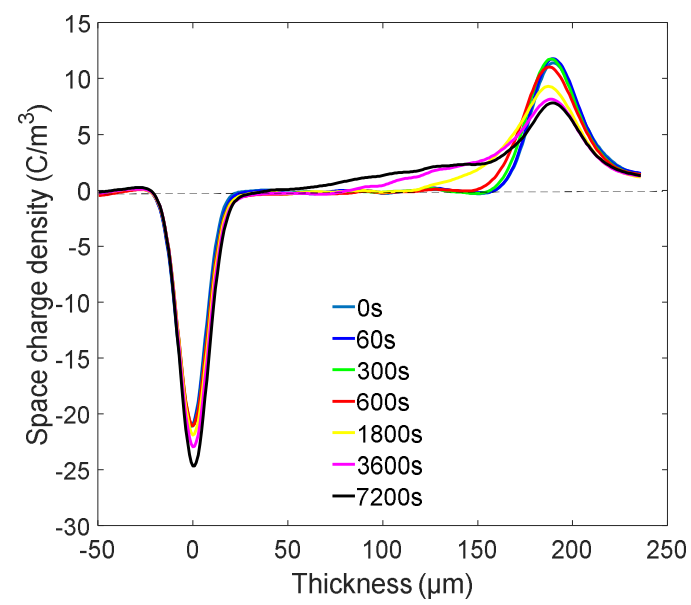

Figure 1. Pulsed electro-acoustic (PEA) measurement results in low density polyethylene (LDPE) film at $20 \mathrm{kV} / \mathrm{mm}$ field under room temperature.

Actually, the charge detected at the interface of electrodes are contributed from the capacitive charge, which induced by the polarization of the dielectric material under a DC field, and the surface charge induced by the accumulated space charge inside the insulation bulk. Generally, the quantity of capacitive charge is much larger than the accumulated charge, which could be a hinderance for observing space charge accumulation. Moreover, the space charge modelling method only simulates the charge dynamics which accumulates inside the bulk. Therefore, in this study, in order to achieve the comparability of the measured data and the simulation results, the capacitive charge at the electrodes has been removed by utilizing the subtraction process proposed by Ning et al. [22]. The charge density after subtraction, $\rho_{a c c}$, can be obtained by applying Equation (1).

$$
\rho_{\text {acc }}(x)=\rho_{\text {test }}(x)-\frac{V_{\text {applied }}}{V_{\text {ref }}} \rho_{\text {ref }}(x)
$$

where $V_{\text {applied }}, V_{\text {ref }}$ are the applied voltage and the reference voltage, respectively, and $\rho_{\text {test }}, \rho_{\text {ref }}$ are the measured charge density and the reference one, respectively. Figure 2a presents the space charge behavior in LDPE sample after the subtraction process, and only the accumulated charge inside the bulk and the induced charge at the electrodes remains. The movement of both positive and negative 
charge carriers can be observed much more clearly. The large positive charge accumulation affected the electric field distribution greatly, as presented in Figure $2 b$. The field strength near the cathode was enhanced from 20 to $24 \mathrm{kV} / \mathrm{mm}$, while it was strongly reduced in the vicinity of anode.

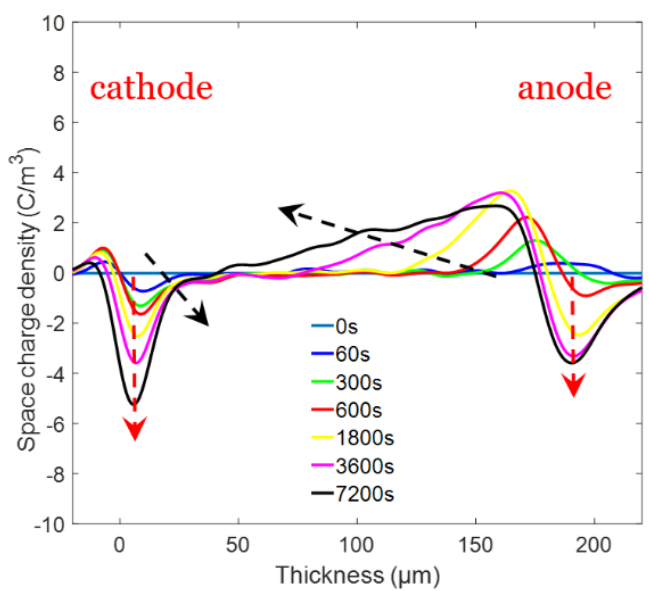

(a)

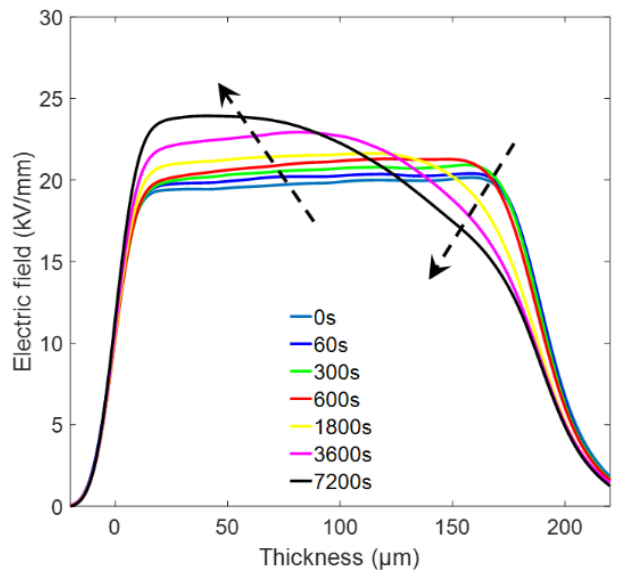

(b)

Figure 2. (a) Space charge behaviour in LDPE film at $20 \mathrm{kV} / \mathrm{mm}$ field under room temperature (capacitive charge removed). (b) Electric field distribution over the insulation thickness of the LDPE.

The space charge profiles in the fresh XLPE sample is shown in Figure 3. After removing the capacitive charge, the hetero charge formation in the fresh XLPE can be clearly observed, as given in Figure 4a. The accumulation of hetero charge at both electrodes increased with time. It seems that the amount of positive charge carriers near the cathode is larger than the amount of negative ones at the adjacent electrode. Due to the presence of hetero charge accumulation, the field distribution was deformed greatly, as shown in Figure $4 \mathrm{~b}$. After $2 \mathrm{~h}$ poling, the maximum electric field location was in the vicinity of cathode, and the field strength in the middle of the dielectric was strongly reduced.

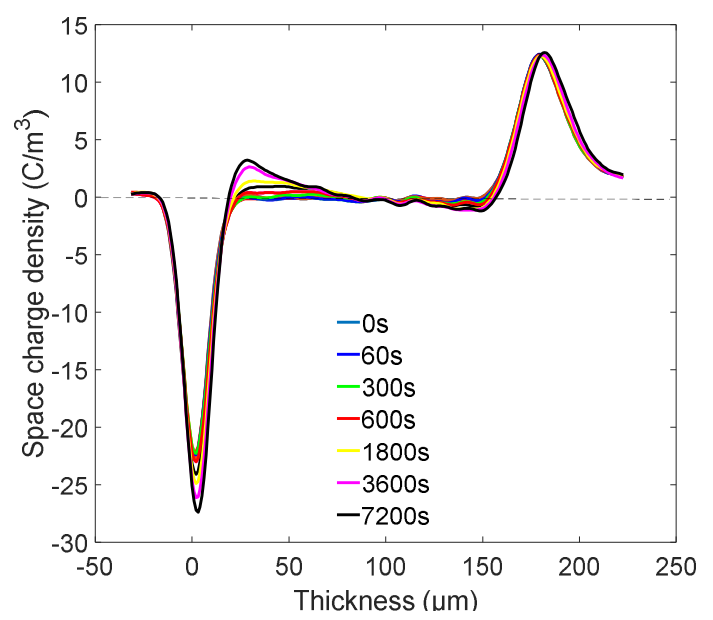

Figure 3. PEA measurement data in fresh cross-linked polyethylene (XLPE) samples at $20 \mathrm{kV} / \mathrm{mm}$ field under room temperature. 


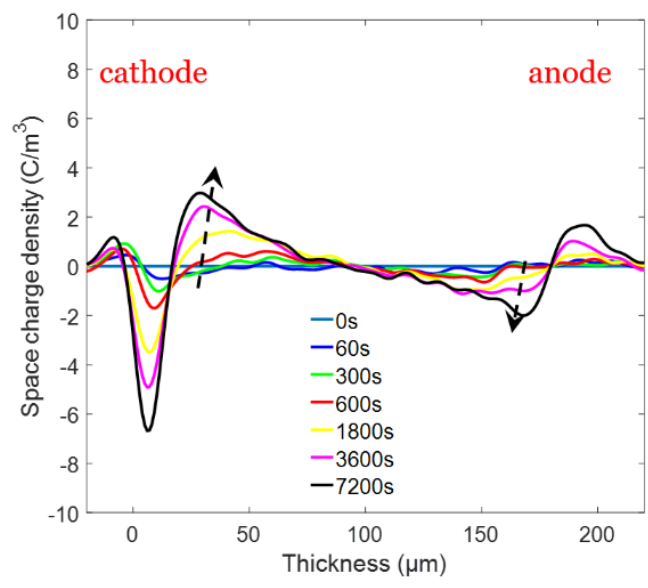

(a)

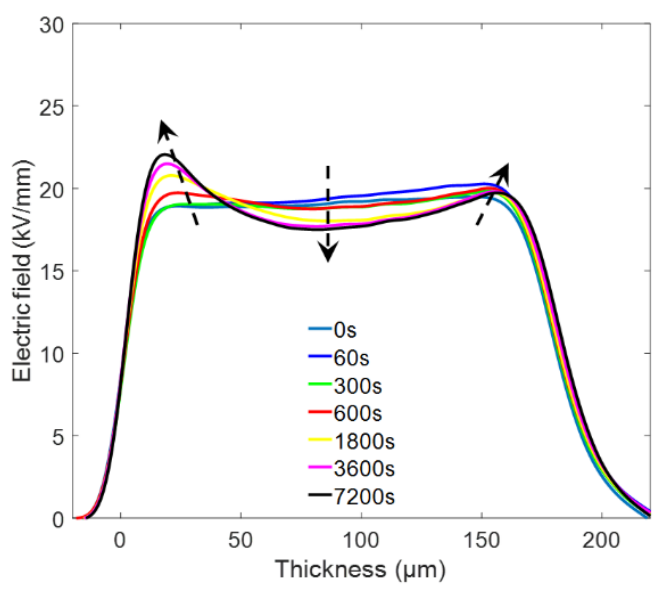

(b)

Figure 4. (a) Space charge behaviour in fresh XLPE samples at $20 \mathrm{kV} / \mathrm{mm}$ field (capacitive charge removed). (b) Electric field distribution across the fresh XLPE film.

Figure 5 shows the space charge profiles in the degassed XLPE sample at the room temperature. Only very limited charge accumulation can be detected inside the insulation bulk. After removing the capacitive charge, a small amount of negative charge carriers can be detected near the cathode, as shown in Figure 6a. The homo charges accumulated near the cathode can be considered to be generated from charge injection, and no obvious charge accumulation can be detected near the anode. Due to the limited charge accumulation inside the bulk, the electric field distribution was nearly unchanged, as presented in Figure 6b.

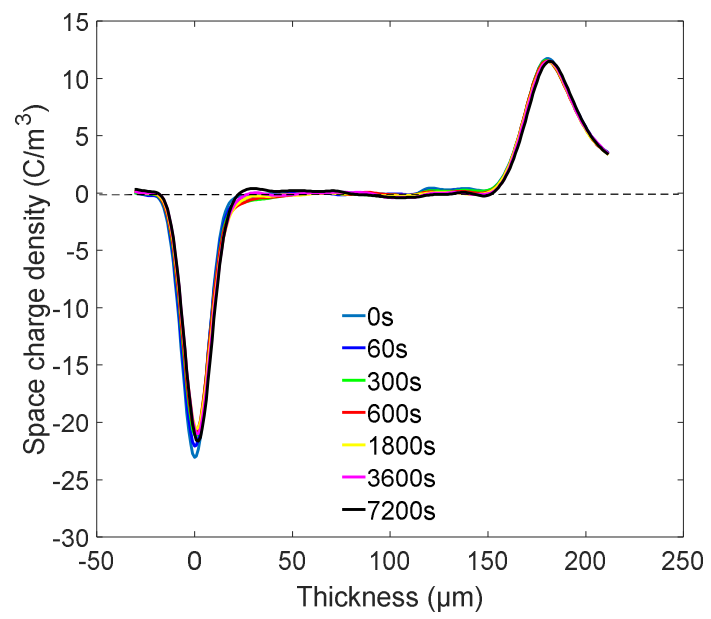

Figure 5. PEA measurement results in the degassed XLPE samples at $20 \mathrm{kV} / \mathrm{mm}$ field under room temperature.

From the experimental observations, we can draw the conclusion that, at the applied field of $20 \mathrm{kV} / \mathrm{mm}$, homo charge distribution is dominant in LDPE, and the amount of accumulated charge (mainly positive charges) increased with time. This kind of profile is always measured for pure LDPE under low applied voltage at room temperature [23]. For fresh XLPE samples, the considerable accumulation of hetero charges can be detected at both electrodes. The increased amount of hetero charges enhanced the electric field near the cathode effectively, which could lead to more charge carriers being injected from the electrode. It should be also noted that the negative charge carriers behaved differently from the positive ones, and this is considered to be the result of the combined effects of the injection and ionization processes working together. Compared to the measured data of the degassed XLPE samples under the same applied field, it can be ensured that the hetero charge 
formation is mainly due to the ionization process of the crosslinking by-products. The detected limited charge accumulation in the degassed XLPE indicates that there might be still some residual crosslinking by-products inside the bulk.

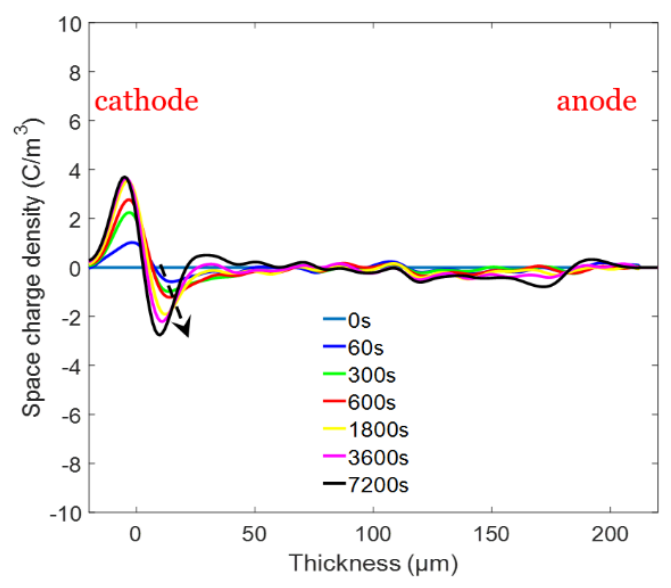

(a)

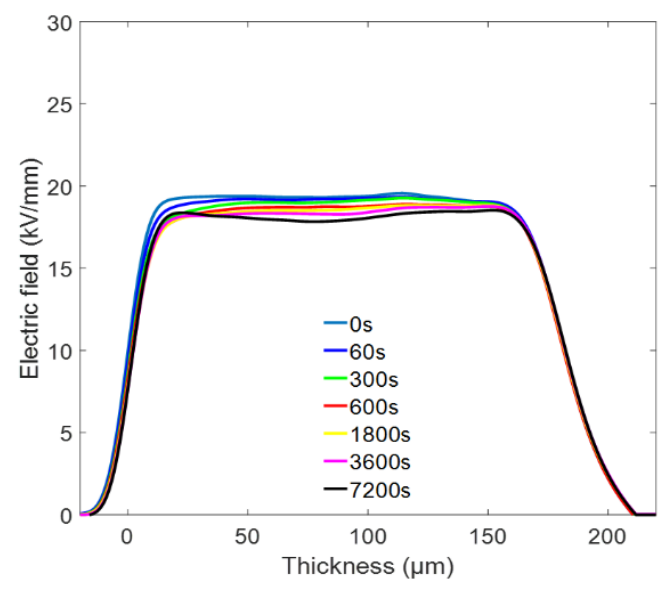

(b)

Figure 6. (a) Space charge behaviour in degassed XLPE samples at $20 \mathrm{kV} / \mathrm{mm}$ field (capacitive charge removed). (b) Electric field distribution across the degassed XLPE film.

\section{Space Charge Modelling Methods}

In this section, a bipolar charge transport model and the modified model, by considering the ionization of dissociable species, will be employed to simulate the space charge dynamics in LDPE and XLPE samples separately. The electrical and thermal conditions are set to be same as the experiments. Both models are implemented by using COMSOL Multiphysics, which is a general-purpose simulation software using finite element analysis method (FEM) and applied for a number of scientific fields [24]. The "Transport of Diluted Species", the "Heat Transfer in Solids", and the "Electrostatics" modules have been applied to simulate the charge dynamics migrating in electric field. The thickness of the sample is divided into 180 divisions equally.

\subsection{Bipolar Charge Transport Model}

The bipolar charge transport theory features the injection of both positive and negative charge carriers from the interfaces of electrode/insulation when the applied DC field exceeds the threshold. Under the force of applied electric field, the injected positive and negative charge carriers (holes and electrons) move towards the opposite electrode, as shown in Figure 7. Due to the existence of the localized energy states in the band gap of the dielectric material, the injected charge carriers can be captured by these "traps", and the trapped charges can also escape to form mobile ones. Moreover, these charge carriers with opposite polarity can possibly recombine, resulting in electroluminescence [25]. Three space and time dependent equations, the Poisson's equation, the continuity equation, and the transport equation, are presented to describe the charge behaviour in this model, as follows:

$$
\begin{gathered}
\frac{\partial E(x, t)}{\partial x}=\frac{\rho(x, t)}{\varepsilon_{0} \varepsilon_{r}} \\
\frac{\partial n_{a \mu, a t}(x, t)}{\partial t}+\frac{\partial\left(j_{a}\right)}{\partial x}-D_{f} \frac{\partial^{2} n_{a u, a t}}{\partial_{x^{2}}}=s_{a \mu, a t}(x, t) \\
j_{a}(x, t)=\mu_{a}(x, t) n_{a \mu}(x, t) E(x, t)-D_{f} \frac{\partial n_{a}(x, t)}{\partial x}
\end{gathered}
$$


where $j_{a}$ is the conduction current density; $n_{a}$ is the charge carrier density; a refers to the type of charge, and the subscript $\mu$ or $t$ refers respectively to mobile or trapped charge; $E$ is the electric field, $\rho$ is the net charge density. $D_{f}$ is the diffusion coefficient, which is assumed to describe the natural motion of chemical species based on the space gradient of particle concentration; $\varepsilon_{0}$ is the vacuum permittivity and $\varepsilon_{r}$ is the relative dielectric permittivity for polyethylene. The term $s$ is the source term, which encompasses the changes in local charge density by processes other than transport, such as the internal generation of charges. For mobile electrons, $s_{e \mu}$ can be expressed as:

$$
s_{e \mu}=\frac{\partial_{n_{e \mu}}}{\partial t}=-S_{1} n_{h t} n_{e \mu}-S_{3} n_{h \mu} n_{e \mu}-B_{e} n_{e \mu}\left(1-\frac{n_{e t}}{n_{o e t}}\right)+D_{e} n_{e t}
$$

where $S_{i}$ are the recombination coefficients; $B_{e}$ and $D_{e}$ are the trapping and detrapping coefficients, respectively. In this model, the charge generation in the clean LDPE is considered from injection at the electrodes following the Schottky law, and a threshold electric field $(10 \mathrm{kV} / \mathrm{mm})$, at which the charge injection takes place, was introduced [26]. Based on many experimental observations in LDPE, no evidence of hetero charge accumulation is found, so it seems not necessary to introduce extraction barriers to simulate charge dynamics in LDPE [27]. A constant effective mobility of charge carriers has been applied to describe the charge migration process under the constant DC field at room temperature. The parameters used in the bipolar charge transport theory are given in Table 1, and these have been optimized from the space charge and conduction current measurements achieved on flat LDPE samples [28].

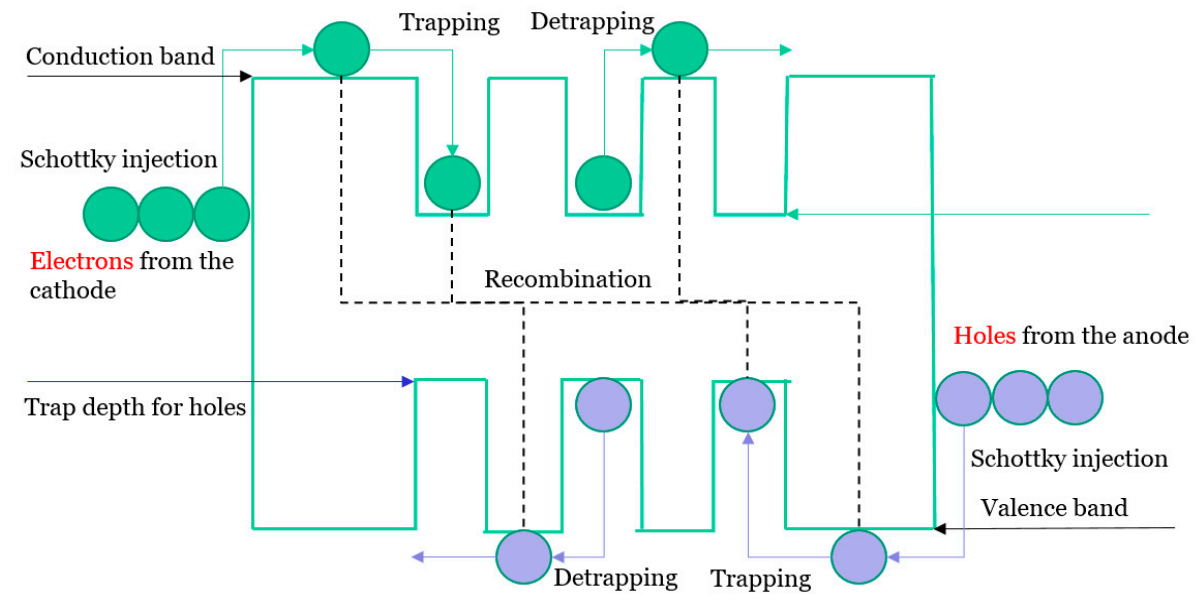

Figure 7. Schematic representation of the bipolar charge transport theory [29].

Table 1. Parameters used for simulating space charge in LDPE [28].

\begin{tabular}{cccc}
\hline \multirow{2}{*}{ Symbol } & & Value & Unit \\
\hline \multirow{2}{*}{ Injection barrier height } & for electrons & 1.27 & $\mathrm{eV}$ \\
& for holes & 1.20 & $\mathrm{eV}$ \\
\hline \multirow{2}{*}{ Trapping coefficient } & for electrons & 0.05 & $\mathrm{~s}^{-1}$ \\
& for holes & 0.05 & $\mathrm{~s}^{-1}$ \\
\hline \multirow{2}{*}{ Mobility } & for electrons & $4 \times 10^{-15}$ & $\mathrm{~m}^{2} /(\mathrm{V} \cdot \mathrm{s})$ \\
& for holes & $1 \times 10^{-14}$ & $\mathrm{~m}^{2} /(\mathrm{V} \cdot \mathrm{s})$ \\
\hline \multirow{2}{*}{ Trap density } & for electrons & 100 & $\mathrm{C} / \mathrm{m}^{3}$ \\
& for holes & 100 & $\mathrm{C} / \mathrm{m}^{3}$ \\
\hline \multirow{2}{*}{ Detrapping barrier height } & for electrons & 0.95 & $\mathrm{eV}$ \\
& for holes & 0.95 & $\mathrm{eV}$ \\
\hline \multirow{2}{*}{ Recombination rate } & $\mathrm{S}_{1}, \mathrm{~S}_{2}, \mathrm{~S}_{3}$ & $4 \times 10^{-3}$ & $\mathrm{~m} 3 /(\mathrm{C} \cdot \mathrm{s})$ \\
& $\mathrm{S}_{0}$ & 0 & $\mathrm{~m} 3 /(\mathrm{C} \cdot \mathrm{s})$ \\
\hline
\end{tabular}


The simulated homo charge distribution in LDPE samples is presented in Figure 8a. After the application of DC voltage, much more holes are injected into the insulation bulk than the electrons, and the migration of holes is much deeper, compared to the accumulation of electrons in the vicinity of cathode. This is because the assumptions of lower injection barrier height and higher charge mobility for holes, as shown in Table 1. It can be observed that the positive charge carriers distributed extensively in the LDPE sample after $2 \mathrm{~h}$ polarization, while the penetration of negative ones is still very limited. The presence of the large amount of accumulated homo charges will distort the field distribution severely, as shown in Figure 8b. Following the movement of positive and negative charge carriers, the electric field strength near the anode decreases, but increases gradually at the cathode. Finally, the maximum electric field locates near the cathode and reaches a value of $24.5 \mathrm{kV} / \mathrm{mm}$, which is consistent with the experimental observation given in Figure $2 b$.

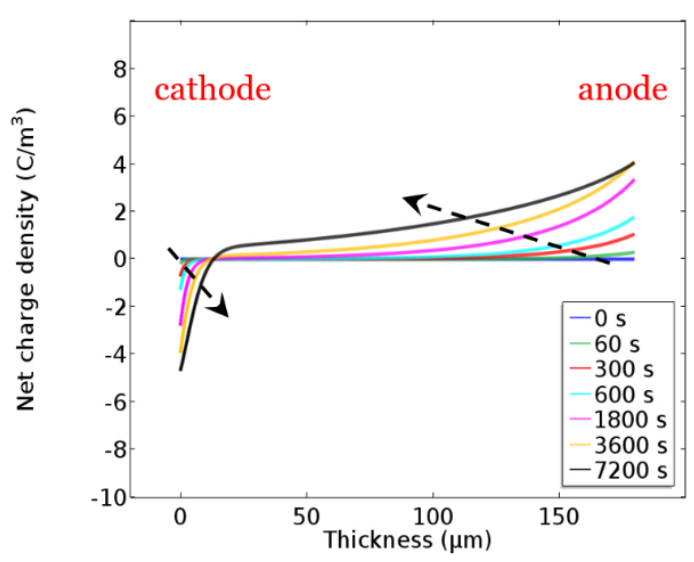

(a)

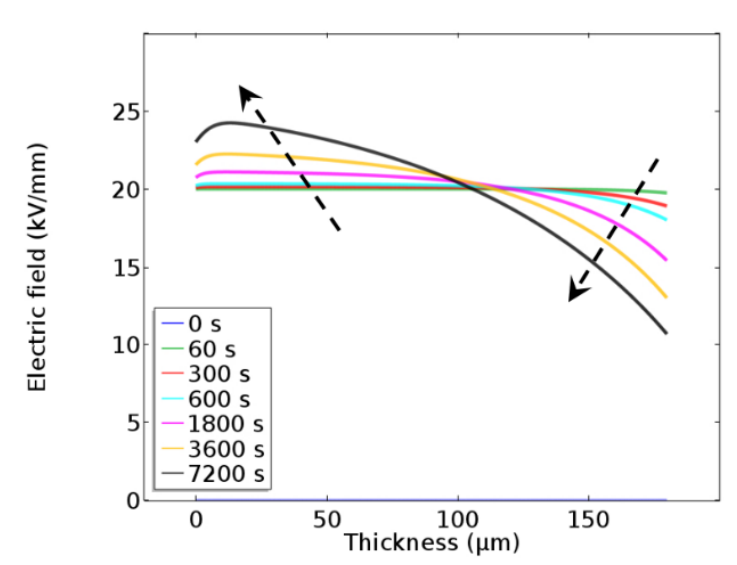

(b)

Figure 8. (a) The simulated space charge distribution in the LDPE sample at $20 \mathrm{kV} / \mathrm{mm}$ field. (b) Calculated electric field distribution over the thickness of LDPE film.

\subsection{Modified Model by Considering Ionization}

The space charge distributions in the fresh and degassed XLPE samples indicate that the formation of hetero charges mainly depends on ionization of impurities, like additives and crosslinking by-products. In order to simulate the space charge behavior in XLPE by considering the charge carriers generated from the dissociation of impurities, some assumptions have been made as follows. For the sake of simplification, only one type of uniformly distributed neutral ion-pair $A B$ is assumed to be the impurity within the insulation, instead of considering the various kinds of crosslinking by-products and antioxidants. The neutral ion-pairs can be thermally activated and dissociated into positive ions, $A^{+}$, and negative ones, $B^{-}$, under the influence of the electrical field, as presented in the following equation:

$$
A B \underset{\text { recombination }}{\stackrel{\text { dissociation }}{\longrightarrow}} A^{+}+B^{-}
$$

According to the Onsager mechanism, the dissociation rate of the neutral ion-pairs is field-dependent as dissociation increases by the factor of $f(E, T)$, while the recombination rate is independent to the electric field because the derivation of recombination is equivalent to the Langevin equation [30]. The electric field dependence of dissociation rate can be expressed as:

$$
D_{d}(E, T)=D_{d}^{0} f(E, T)
$$


where $D_{d}^{0}$ is the dissociation constant at zero electric field, and $f(E, T)$ is the field and temperature dependent function, which is defined as:

$$
f(E, T)=\frac{I_{1}(4 b)}{2 b} \text { where } b=\sqrt{\frac{q^{3}|E|}{16 \pi \varepsilon_{0} \varepsilon_{r} k^{2} T^{2}}}
$$

where $I_{1}$ is the modified Bessel function of the first kind and $k$ is the Boltzmann's constant. The initial neutral ion-pair concentration is set to be $N_{0}$. Under the applied DC field, the generated positive and negative ions will be attracted to the opposite polarity electrode, forming the ionic conduction current. Following the assumptions made in the charge transport theory, it is also presumed that the generated positive and negative ions have the possibility to be trapped, detrapped, and recombined with inverse polarity ions. Therefore, the source term of mobile negative ions $s_{n \mu}$ can be expressed as:

$$
s_{n \mu}=\frac{\partial n_{n \mu}}{\partial t}=-S_{i o n 1} n_{p t} n_{n \mu}-S_{i o n 3} n_{p \mu} n_{n \mu}-B_{n} n_{n \mu}\left(1-\frac{n_{n t}}{n_{o n t}}\right)+D_{n} n_{n t}+D_{d} N(x)
$$

where $B_{n}, D_{n}$ are the trapping and detrapping coefficients, respectively, and the recombination coefficients $S_{\text {ion } i}$ are of the Langevin form and function of the ionic charge carrier mobility.

$$
S_{\text {ion } p, n}=\frac{\mu_{p, n}}{\varepsilon_{0} \varepsilon_{r}}
$$

The concentration of the residual neutral ion-pairs varies with the dissociation and recombination processes; thus, the variation of the impurity density can be expressed as:

$$
\Delta N(x)=\frac{\partial N(x)}{\partial t}=S_{i o n 0} n_{n t} n_{p t}+S_{i o n 1} n_{n \mu} n_{p t}+S_{i o n 2} n_{n t} n_{p \mu}+S_{i o n 3} n_{n \mu} n_{p \mu}-D_{d} N(x)
$$

In this modified model, nine kinds of species are considered within the insulation bulk. In the case of positive/negative ions, it is difficult for them to be extracted at the electrodes due to the mass. Therefore, in this model, the ions are considered to be totally blocked after reaching the opposite polarity electrode. However, it should be noted that, without considering any diffusion coefficients, the accumulation of ionic charges (trapped or mobile) at the interface of electrodes/dielectric can lead to numerical errors. Table 2 gives the parameters used in the modified model for simulating space charge behavior in the XLPE samples. The initial ion-pair concentration in fresh XLPE samples is estimated from the proportion of crosslinking by-products in XLPE [9]. Although it has been observed that the space charge accumulation in LDPE behaves differently from that in XLPE, and the explanation could be addressed to the introduction of antioxidant and crosslinking, which increases the deep trap density and introduces deeper traps [31]. However, in this initial model, the parameters relating to the holes and electrons remain unchanged, following the values given in Table 1, in order to evaluate if the dissociation can be accounted for the hetero charge formation in XLPE.

The simulated space charge profiles in the fresh XLPE samples are given in Figure 9a, and it should be noted that the charge distribution results from both the charge carriers injected from the electrodes and the dissociated ionic charge carriers. Under the applied field, the dissociated positive and negative ions moved towards the opposite polarity electrode, forming hetero charge accumulation. The amount of accumulated hetero charges at both electrodes increased with time. Compared with the accumulation of negative charge carriers near the anode, the hetero charge formation at the cathode was more considerable, and this is not only due to the assumption of a higher mobility of positive ions, but also to the contribution of the holes injected from the electrode, which reduce the amount of negative charge carriers presented near the anode. Figure $9 \mathrm{~b}$ shows the electrical field distribution distorted by the presence of hetero charge accumulation. The field strength was enhanced near both electrodes, but decreased in the middle part of the insulation. 
Table 2. Parameters used for simulating space charge in XLPE at room temperature.

\begin{tabular}{cccc}
\hline \multicolumn{1}{c}{ Symbol } & Value & Unit \\
\hline \multirow{2}{*}{ Initial ion-pair concentration } & $\begin{array}{c}\text { for fresh XLPE } \\
\text { for degassed XLPE }\end{array}$ & 40 & $\mathrm{~mol} / \mathrm{m}^{3}$ \\
& for ion-pairs & $6.1 \times 10^{-11}$ & $\mathrm{sol}^{-1}$ \\
\hline Dissociation rate at zero field & for positive ions & 0.1 & $\mathrm{~s}^{-1}$ \\
\hline \multirow{2}{*}{ Trapping coefficient } & for negative ions & 0.1 & $\mathrm{~s}^{-1}$ \\
\hline \multirow{2}{*}{ Mobility } & for positive ions & $2 \times 10^{-16}$ & $\mathrm{~m}^{2} /(\mathrm{V} \cdot \mathrm{s})$ \\
& for negative ions & $1 \times 10^{-16}$ & $\mathrm{~m}^{2} /(\mathrm{V} \cdot \mathrm{s})$ \\
\hline \multirow{2}{*}{ Trap density } & for positive ions & 100 & $\mathrm{C} / \mathrm{m}^{3}$ \\
& for negative ions & 100 & $\mathrm{C} / \mathrm{m}^{3}$ \\
\hline \multirow{2}{*}{ De-trapping barrier height } & for positive ions & 0.95 & $\mathrm{eV}$ \\
& for positive ions & 0.95 & $\mathrm{eV}$ \\
\hline Diffusion coefficient & for ions, holes and electrons & $1 \times 10^{-12}$ & $\mathrm{~m}^{2} / \mathrm{s}$ \\
\hline
\end{tabular}

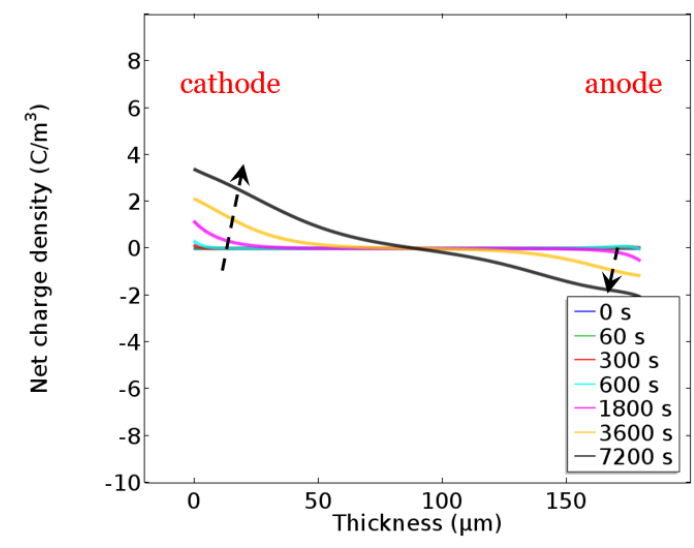

(a)

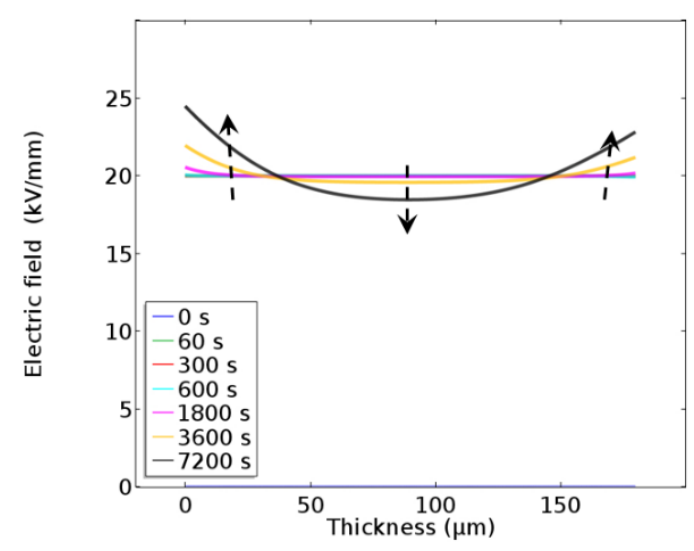

(b)

Figure 9. (a) Simulated space charge accumulation evolving with time in fresh XLPE samples at $20 \mathrm{kV} / \mathrm{mm}$ field under room temperature. (b) Calculated electric field distribution across the fresh XLPE sample.

The space charge distribution in the degassed XLPE samples are obtained by reducing the concentration of dissociable by-products, as shown in Figure 10a. The presence of space charge accumulation is reduced greatly, leading to an almost unchanged electric field distribution, as presented in Figure 10b. Since thermal treatment can expel most volatile species, such as crosslinking by-products, the initial neutral ion-pairs concentration in degassed XLPE sample is much less. It can be observed that negative charge carriers slightly accumulate in the vicinity of cathode, while very limited charges occur near the anode. It can be argued that the injection of electrons from the cathode becomes more dominated in charge accumulation, when the impact of dissociation is reduced by eliminating the amount of impurities.

Generally, the outputs from the modelling methods are comparable with the experimental observations. The homo charge accumulation inside the LDPE can be simulated by the bipolar charge transport model with the appropriate parameters setting, and both the space charge and field distribution fits the experimental results well, including the charge movement and field variation with time. With the modified model, by considering the dissociation of impurities, the hetero charge formation at both electrodes can be simulated in XLPE under the DC field. The location and the amount of accumulated charges within the bulk are quantitatively consistent with the experimental observations. It should be clarified that part parameters used for the modified model are estimated following the 
parameter setting of the bipolar charge transport model and fitting the experimental results. The range of the parameters for simulating the dissociation process still need to be further validated.

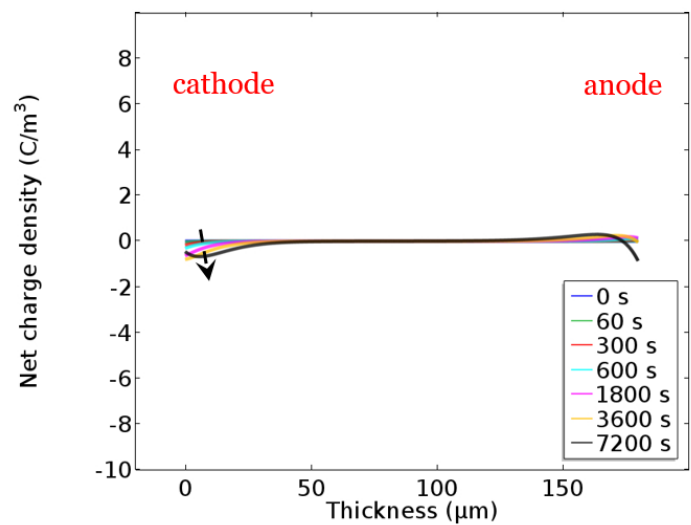

(a)

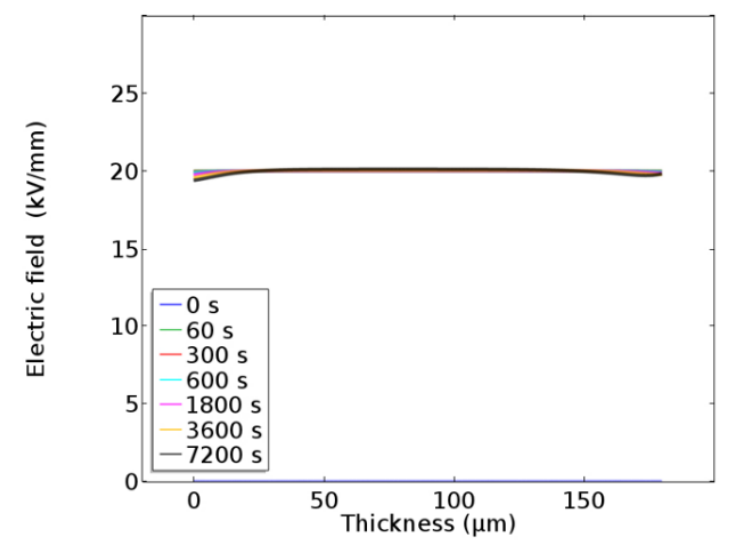

(b)

Figure 10. (a) Space charge accumulation simulated by the modified model in degassed XLPE sample at $20 \mathrm{kV} / \mathrm{mm}$ field. (b) Electric field distribution across the degassed XLPE film.

\section{Discussion}

The simulation results provided in last section indicates the modified model can show appropriate features on space charge behavior, especially the formation of hetero charge in XLPE. This modified model can not only contribute to simulate dynamic charge and field distribution in XLPE, but also assist in understanding the mechanisms of charge generation and transport. Even with the parameters used for LDPE and the estimated parameters for dissociation, the amount of hetero charges accumulated with time at both electrodes can be observed by this preliminary model, and both the charge movement and field distribution in fresh/degassed XLPE are consistent with the experimental observations. Thus, it seems that the modified model can be potentially applied to simulate space charges in cable geometry and to calculate the electric field distribution along the insulation radial thickness. However, in practical loaded DC cable applications, the space charge build-up can be affected greatly by the temperature gradient over the insulation thickness and the divergent field, due to the cable geometry factor. Therefore, in this section, the restrictions and the potential improvements of this model will be discussed.

Based on the experimental work published in [5,32], the space charge accumulation in an XLPE power cable can be greatly affected by the temperature gradient under DC field. The hetero charge formation at both electrodes performed differently with or without the temperature gradient, as shown in Figure 11. It seems that the presence of temperature gradient can not only affect the origins of space charge (injection or dissociation), but also assist the charge transportation. In the further development of this modified model, thermal and electrical effects on the charge generation and conduction need to be taken into account. For example, the conduction of electrons/holes/ions can be described by a hopping type mobility as a function of both temperature and field, instead of using a constant effective mobility.

One restriction of this initial model is that only one type of impurity which can be dissociated into positive and negative ions is considered, and the concentration level of the impurity is assumed to distribute uniformly in the XLPE. However, the field-assisted thermal ionization of impurity molecules would also generate positive ions and electrons. If the electrons that did not recombine with positive ions or holes, they would be extracted at the anode or deeply trapped in the bulk [6]. It is also reported that the concentrations of crosslinking by-products in different regions along the cable radius are different from each other, probably due to the degassing stage in cable manufacture [33]. Therefore, some updates can be applied on this modified model, such as introducing another kind of impurity species, considering the interactions between the generated ions/electrons and the injected charge 
carriers. Furthermore, a concentration gradient of impurities in the thick insulation layer of cables should be also taken into account. In addition to these, the properties of the semicon/insulation interface plays a major role in charge injection and accumulation, as the contained impurities may diffuse into the bulk, assisting the formation of hetero charges [34].

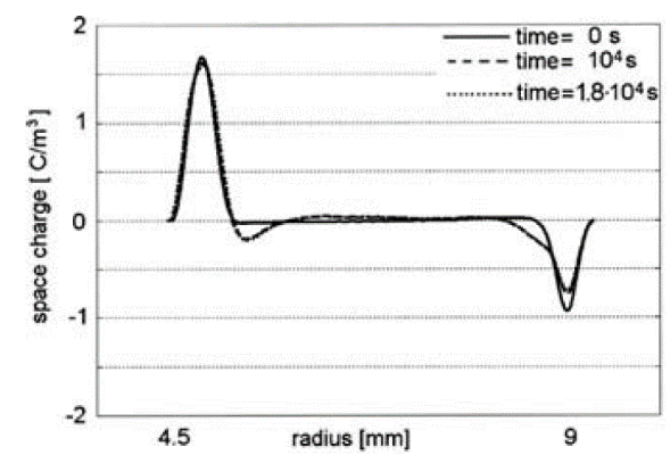

(a)

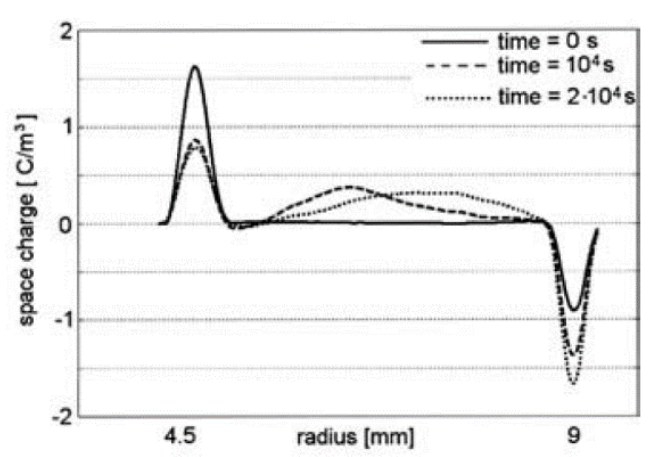

(b)

Figure 11. Space charge profiles from tests on XLPE MV-size cables with an average field of $20 \mathrm{kV} / \mathrm{mm}$. (a) no temperature gradient. (b) with a temperature gradient of $20^{\circ} \mathrm{C}$ [5].

In general, this preliminary model can be further improved by considering the thermal effects on the mechanisms of charge generation and charge transport, as well as by taking into account the concentration gradient of dissociable impurities, in order to predict the charge behavior and field distribution in HVDC extruded cables in practical situation. Additionally, the simulation results still need to be further validated by the space charge measurement of XLPE cables under the temperature gradient, which is being prepared in the Tony Davis High Voltage Laboratory.

\section{Conclusions}

Under a $20 \mathrm{kV} / \mathrm{mm}$ DC field, considerable hetero charge formation was observed in fresh XLPE samples, and only limited charge accumulation could be detected in the degassed XLPE samples, indicating the ionic charges play an important role in space charge behavior of XLPE. Compared with the experimental observations, the homo charge distribution simulated by the bipolar charge transport model could fit the measured data well. A modified model, by considering the dissociation of impurities, was employed to simulate the space charge behavior in XLPE. The features of hetero charge formation could be predicted efficiently by this modified model, and both the space charge and field distribution were consistent with the experimental results.

The potential optimizations of this preliminary model were discussed based on the practical cable application. It is suggested that the concentration gradient of impurities should be taken into account, and the parameterization of XLPE is still needed.

Author Contributions: Conceptualization, Y.Z. and G.C.; methodology, Y.Z.; software, Y.Z.; writing-original draft preparation, Y.Z.; writing-review and editing, G.C. and M.H.; discussion and funding acquisition, L.P., X.Z., S.W. and J.L.; supervision, G.C. All authors have read and agreed to the published version of the manuscript.

Funding: This research was funded by State Grid Shaanxi Electric Power Company, China.

Acknowledgments: The authors are appreciated for the financial support from the technology project of State Grid Shaanxi Electric Power Company [5226KY16001G] and [2018GY-001].

Conflicts of Interest: The authors declare no conflict of interest.

\section{References}

1. Mazzanti, G.; Marzinotto, M. Extruded Cables for High-Voltage Direct-Current Transmission: Advances in Research and Development; John Wiley \& Sons: Hoboken, NJ, USA, 2013. 
2. Heyman, O.; Weimers, L.; Bohl, M.-L. HVDC-A key solution in future transmission systems. In Proceedings of the World Energy Congress-WEC, Montreal, QC, Canada, 12-16 September 2010; pp. 12-16.

3. Jeroense, M. HVDC, the Next Generation of Transmission: Highlights with Focus on Extruded Cable Systems. IEEJ Trans. Electr. Electron. Eng. 2010, 5, 400-404. [CrossRef]

4. Fabiani, D.; Montanari, G.; Laurent, C.; Teyssedre, G.; Morshuis, P.; Bodega, R.; Dissado, L.; Campus, A.; Nilsson, U. Polymeric HVDC Cable Design and Space Charge Accumulation. Part 1: Insulation/Semicon Interface. IEEE Electr. Insul. Mag. 2007, 23, 11-19. [CrossRef]

5. Fabiani, D.; Montanari, G.; Laurent, C.; Teyssedre, G.; Morshuis, P.; Bodega, R.; Dissado, L. HVDC Cable Design and Space Charge Accumulation. Part 3: Effect of Temperature Gradient [Feature article]. IEEE Electr. Insul. Mag. 2008, 24, 5-14. [CrossRef]

6. Hanley, T.; Burford, R.; Fleming, R.; Barber, K. A general review of polymeric insulation for use in HVDC cables. IEEE Electr. Insul. Mag. 2003, 19, 13-24. [CrossRef]

7. Mazzanti, G.; Montanari, G.; Dissado, L. Electrical aging and life models: The role of space charge. IEEE Trans. Dielectr. Electr. Insul. 2005, 12, 876-890. [CrossRef]

8. Huang, X.; Fan, Y.; Zhang, J.; Jiang, P. Polypropylene based thermoplastic polymers for potential recyclable HVDC cable insulation applications. IEEE Trans. Dielectr. Electr. Insul. 2017, 24, 1446-1456. [CrossRef]

9. Andrews, T.; Hampton, R.; Smedberg, A.; Wald, D.; Waschk, V.; Weissenberg, W. The role of degassing in XLPE power cable manufacture. IEEE Electr. Insul. Mag. 2006, 22, 5-16. [CrossRef]

10. Fu, M.; Chen, G.; Dissado, L.; Fothergill, J. Influence of thermal treatment and residues on space charge accumulation in XLPE for DC power cable application. IEEE Trans. Dielectr. Electr. Insul. 2007, 14, 53-64. [CrossRef]

11. Rizzo, G.; Romano, P.; Imburgia, A.; Ala, G. Review of the PEA Method for Space Charge Measurements on HVDC Cables and Mini-Cables. Energies 2019, 12, 3512. [CrossRef]

12. Takeda, T.; Hozumi, N.; Suzuki, H.; Okamoto, T. Factor of Hetero Space Charge Generation in XLPE under DC Electric Field of 20kV/mm. IEEJ Trans. Fundam. Mater. 1997, 117, 915-921. [CrossRef]

13. Sekii, Y.; Ohbayashi, T.; Uchimura, T.; Hukuyama, T.; Maeno, T. A study on the space charge formation in XLPE. In Proceedings of the 2001 Annual Report Conference on Electrical Insulation and Dielectric Phenomena (Cat. No.01CH37225), Kitchener, ON, Canada, 14-17 October 2001; pp. 469-472.

14. Hussin, N.; Chen, G. Analysis of space charge formation in LDPE in the presence of crosslinking byproducts. IEEE Trans. Dielectr. Electr. Insul. 2012, 19, 126-133. [CrossRef]

15. Montanari, G.; Fabiani, D.; Dissado, L.A. A new conduction phenomenon observed in polyethylene and epoxy resin: Ultra-fast soliton conduction. J. Polym. Sci. Part B 2011, 49, 1173-1182. [CrossRef]

16. McAllister, I.; Crichton, G.; Pedersen, A. Charge accumulation in DC cables: A macroscopic approach. In Proceedings of the 1994 IEEE International Symposium on Electrical Insulation, Pittsburgh, PA, USA, 5-8 June 2002; pp. 212-216.

17. Zhan, Y.; Chen, G.; Hao, M.; Pu, L.; Zhao, X.; Sun, H.; Wang, S.; Guo, A.; Liu, J. Comparison of two models on simulating electric field in HVDC cable insulation. IEEE Trans. Dielectr. Electr. Insul. 2019, 26, 1107-1115. [CrossRef]

18. Alison, J.M.; Hill, R.M. A model for bipolar charge transport, trapping and recombination in degassed crosslinked polyethene. J. Phys. D 1994, 27, 1291-1299. [CrossRef]

19. Fu, M.; Hou, S.; Liu, T.; Lv, Z.; Wu, K.; Wang, Y.; Wang, X. Mechanism of space charge accumulation in crosslinked polyethylene under temperature gradient. In Proceedings of the 2015 IEEE 11th International Conference on the Properties and Applications of Dielectric Materials (ICPADM), Sydney, Australia, 19-22 July 2015; pp. 356-359.

20. Le Roy, S.; Teyssedre, G.; Laurent, C. Modelling space charge in a cable geometry. IEEE Trans. Dielectr. Electr. Insul. 2016, 23, 2361-2367. [CrossRef]

21. Wu, J.; Lan, L.; Li, Z.; Yin, Y. Simulation of space charge behavior in LDPE with a modified of bipolar charge transport model. In Proceedings of the 2014 International Symposium on Electrical Insulating Materials, Niigata, Japan, 1-5 June 2014; pp. 65-68.

22. Liu, N.; Zhou, C.; Chen, G.; Zhong, L. Determination of threshold electric field for charge injection in polymeric materials. Appl. Phys. Lett. 2015, 106, 192901. [CrossRef]

23. Thomas, C.; Teyssedre, G.; Laurent, C. Space-charge dynamic in polyethylene: From dc to ac stress. J. Phys. D 2010, 44, 15401. [CrossRef] 
24. Carlini, M.; McCormack, S.; Castellucci, S.; Ortega, A.; Rotondo, M.; Mennuni, A. Modelling and Numerical Simulation for an Innovative Compound Solar Concentrator: Thermal Analysis by FEM Approach. Energies 2020, 13, 548. [CrossRef]

25. Le Roy, S.; Teyssedre, G.; Segur, P.; Laurent, C. Modelling of space charge, electroluminescence and current in low density polyethylene under DC and AC field. In Proceedings of the 17th Annual Meeting of the IEEE Lasers and Electro-Optics Society, LEOS, Boulder, CO, USA, 20-20 October 2004; pp. 29-32.

26. Zhan, Y.; Chen, G.; Hao, M. Space charge modelling in HVDC extruded cable insulation. IEEE Trans. Dielectr. Electr. Insul. 2019, 26, 43-50. [CrossRef]

27. Teyssedre, G.; Laurent, C. Charge transport modeling in insulating polymers: From molecular to macroscopic scale. IEEE Trans. Dielectr. Electr. Insul. 2005, 12, 857-875. [CrossRef]

28. Le Roy, S.; Teyssedre, G.; Laurent, C.; Montanari, G.C.; Palmieri, F. Description of charge transport in polyethylene using a fluid model with a constant mobility: Fitting model and experiments. J. Phys. D 2006, 39, 1427-1436. [CrossRef]

29. Laurent, C.; Teyssedre, G.; Le Roy, S.; Baudoin, F. Charge dynamics and its energetic features in polymeric materials. IEEE Trans. Dielectr. Electr. Insul. 2013, 20, 357-381. [CrossRef]

30. Castellanos, A. Electrohydrodynamics; Springer Science \& Business Media: Berlin/Heidelberg, Germany, 1998.

31. Montanari, G.; Laurent, C.; Teyssedre, G.; Campus, A.; Nilsson, U. From LDPE to XLPE: Investigating the change of electrical properties. Part I. space charge, conduction and lifetime. IEEE Trans. Dielectr. Electr. Insul. 2005, 12, 438-446. [CrossRef]

32. Fu, M.; Dissado, L.; Chen, G.; Fothergill, J. Space charge formation and its modified electric field under applied voltage reversal and temperature gradient in XLPE cable. IEEE Trans. Dielectr. Electr. Insul. 2008, 15, 851-860. [CrossRef]

33. Ren, H.; Zhong, L.; Liu, M.; Yu, Q.; Li, Y.; Zhao, W.; Zhou, Z. Effects of degassing duration on space charge and DC conductivity in HVDC XLPE cable insulation. In Proceedings of the 2018 12th International Conference on the Properties and Applications of Dielectric Materials (ICPADM), Xi'an, China, 20-24 May 2018; pp. 124-127.

34. Hao, M.; Fazal, A.; Vaughan, A.S.; Chen, G.; Zhou, Y.; Zhang, C. The impacts of degassing on space charge characteristics and DC conductivity in semicon-bonded XLPE for HVDC cable applications. In Proceedings of the 2016 IEEE Conference on Electrical Insulation and Dielectric Phenomena (CEIDP), Toronto, ON, Canada, 16-19 October 2016; pp. 97-100. 